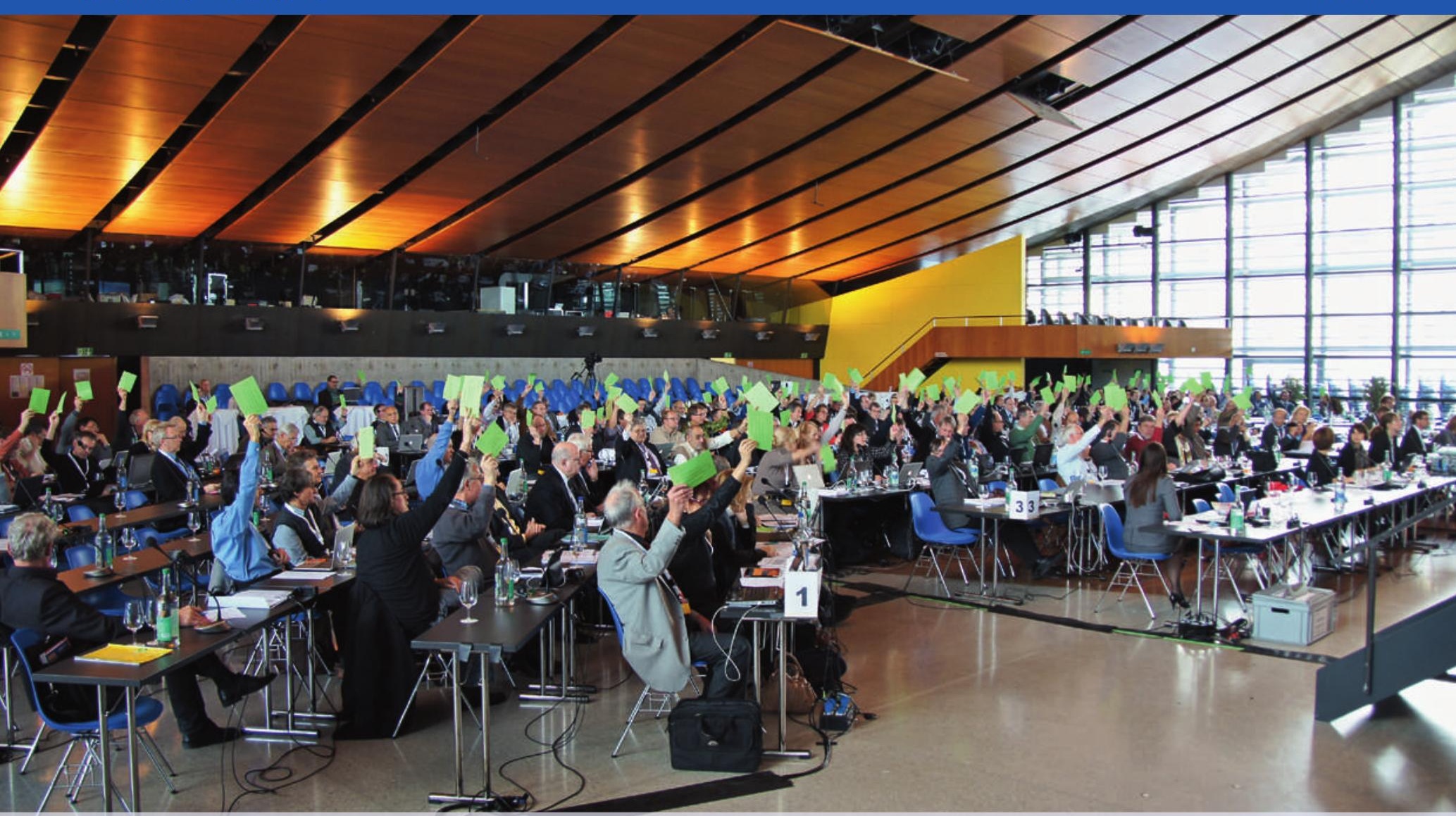

La Chambre médicale compte 200 délégués qui représentent les organisations de base ainsi que les sociétés de discipline médicale.

Ils constituent le «Parlement» de la FMH.

Photos: Christoph Kreyden

Palais des congrès, Bienne, le 6 décembre 2012

\title{
Procès-verbal de la Chambre médicale ordinaire du 6 décembre 2012
}

Anne-Geneviève Bütikofer, secrétaire générale

Un glossaire des abréviations les plus usitées figure à la page 157.

\section{Message de bienvenue, communications, constitution du Bureau}

Une fois le quorum atteint, le président Jacques de Haller souhaite la bienvenue aux délégués et ouvre cette séance ordinaire de la Chambre médicale. Il salue les invités: Roland Schwarz, président de la Commission de gestion (CdG), Silvia Cueni et Maya Schuppli, membres du groupe de travail «Abus à caractère sexuel lors de l'activité médicale» pour le point $n^{\circ} 3$ et Bruno Kesseli, rédacteur en chef du Bulletin des médecins suisses (BMS). Après quelques informations d'ordre organisationnel, le président communique la composition du Bureau de la séance du jour, constitué du président de la FMH Jacques de Haller, des deux vice-présidents Ernst Gähler et Pierre-François Cuénoud, de la Secrétaire générale Anne-Geneviève Bütikofer, ainsi que des scrutateurs Pierre Vallon/Psychiatrie et psychothérapie, Mathias Gugger/Pathologie, Alain Souche/GE, Marcel Stampfli/BE, Jean-Michel Gaspoz/SSMI, Daniel Ackermann/Urologie, Jürg Nadig/Oncologie, MarieChristine Peter-Gattlen/Allergologie, Claude Karen
Gutscher/ASMAC et Daniel Schröpfer/ASMAC.

Le président explique qu'en vertu des dispositions statutaires, il ne sera plus possible de prendre des décisions ou de voter après 17h00. Il indique que l'invitation accompagnée de l'ordre du jour et des annexes a été envoyée par courrier électronique dans les délais, quatre semaines avant la séance de ce jour. Une enveloppe contenant les documents de vote a été remise aux délégués à la Chambre médicale lors de leur arrivée.

\subsection{Ordre du jour}

1.1.1 Point 4 «Abus à caractère sexuel lors de l'activité médicale»

Jacques de Haller passe à la proposition $\mathrm{n}^{\circ} 1$ au point $\mathrm{n}^{\circ}$ 1.1.1 de Christoph Ramstein/VEDAG, qui demande de modifier l'ordre du jour en avançant le point $n^{\circ} 4$ «Abus à caractère sexuel» pour éviter de devoir le traiter dans la précipitation en raison d'un manque de temps. Le président propose à la Chambre médicale d'intervertir les points $n^{\circ} 3$ «Budget» et $n^{\circ} 4$. 
- La proposition $n^{\circ} 1$ relative au point $n^{\circ} 1.1 .1$ de Christoph Ramstein est acceptée à une large majorité.

Le président donne la parole à Emanuel Waeber/FMH qui présente les collaboratrices et collaborateurs entrés au service de la FMH depuis la dernière séance de la Chambre médicale: Olga Pfammatter et Michèle Wyder, collaboratrices de l'ISFM; Yasmine Gautschi, responsable du Secrétariat central; Johnny Graber et Philipp Hofer, développeurs informatiques; Ursina Pally, responsable suppléante du service juridique; Kerstin Rutsch, assistante pour le domaine «Tarifs et conventions pour la médecine ambulatoire en Suisse», et Cornelia Steck au service de communication.

\section{Informations du Comité central, du Secrétariat général, des domaines d'activité et de I'ISFM}

\subsection{Comité central}

\subsubsection{Tarifs et conventions pour la médecine} ambulatoire en Suisse (TARVISION; Masterplan «Médecine de famille»)

Ernst Gähler/CC informe de la situation actuelle dans le domaine tarifaire et précise que les positions se durcissent de plus en plus. Il note de grandes divergences de vue entre les partenaires tarifaires, notamment en ce qui concerne l'interprétation des faits. L'OFSP reproche à la FMH une approche unilatérale dans le projet TARVISION et santésuisse affirme, entre autres, que l'étude sur les revenus montre qu'il n'y a aucun besoin d'agir pour le revenu des médecins, ces derniers ayant amélioré leurs revenus de CHF 220000.- au cours des vingt dernières années,

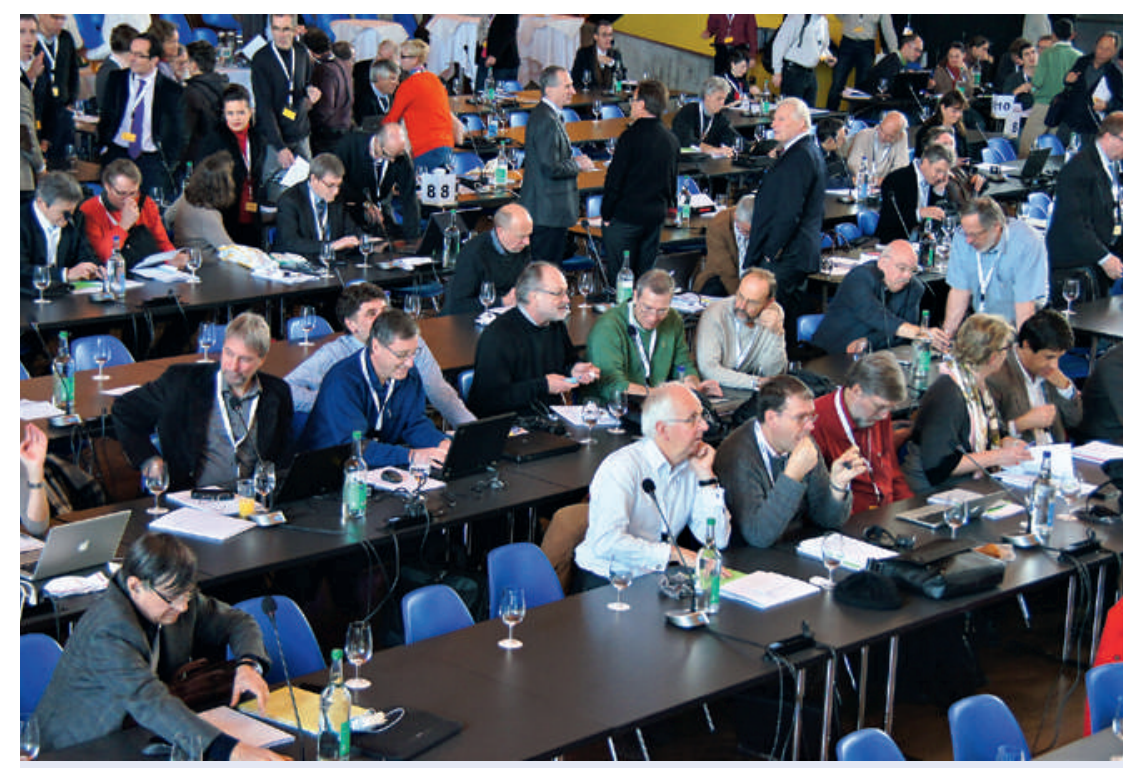

Avant le début de la séance, l'assemblée connaît déjà la même ébullition... ce qui correspond à un taux d'augmentation annuel de $2,5 \%$. Cependant, les chiffres de la FMH qui reposent sur vingt ans de données ROKO, sur dix-huit ans de TARMED et sur deux ans de TARVISION parlent un tout autre langage. En effet, le revenu des médecins n'a augmenté que de CHF 20000.- en vingt ans, soit un taux de $0,6 \%$ par an, alors que parallèlement les dépenses ainsi que les salaires non médicaux ont augmenté massivement. A titre de comparaison, l'administration a enregistré une augmentation de $21,3 \%$, et le domaine social de $22,8 \%$. E. Gähler constate que si on veut parler d'une explosion des coûts, celle-ci concerne les autres et non les médecins.

La révision de la structure tarifaire dans le cadre du projet TARVISION de la FMH se poursuit avec la même intensité. Les sociétés de discipline médicale sont étroitement impliquées dans les travaux. La collaboration avec $\mathrm{H}+$ et la CTM fonctionne de manière constructive. E. Gähler reste confiant et estime que la révision sera achevée d'ici à fin 2013.

\section{La FMH s'engage pour traiter en priorité l'introduction du chapitre 40 dans le TARMED}

La Commission tarifaire de la MFE a terminé ses travaux relatifs au chapitre 40 du TARMED, dédié spécialement aux médecins de premier recours. Les nouveautés les plus importantes sont les positions créées pour les consultations d'urgence; la suppression de la rémunération à demi-tarif des «cinq dernières minutes» (position 00.0030) en faveur d'une rémunération au temps consacré (prestations classiques); la nouvelle valeur intrinsèque désignée par FMH 5+ (auparavant FMH 5) qui sera calculée avec le facteur 1.0 au lieu de 0.905 ; le statut d'organe à trois niveaux (petit, moyen, grand) ainsi que les principes de facturation et les droits acquis. Il s'agit maintenant de trouver la mise en application technique correcte en termes de tarifs et de l'intégrer dans la structure tarifaire. La FMH soutiendra à cet égard la Commission tarifaire de la MFE.

Au sein de la FMH, personne ne conteste le chapitre 40. Mais son financement est remis en cause: la Confédération et santésuisse exigent la neutralité des coûts au cours des prochaines années. La position de la FMH est cependant claire: la neutralité des coûts n'est ancrée dans aucune loi. Par conséquent, ni la MFE, ni la FMH n'approuveront un transfert linéaire des coûts.

La FMH met tout en œuvre pour que le chapitre 40 soit introduit avant la révision globale. Pour cela, il faut que le financement vienne de l'extérieur. Un financement généré par le TARMED, si toutefois cela est possible, ne pourrait être réalisé qu'au terme de la révision globale, à condition que les volumes nécessaires soient libérés. Il est nécessaire de traiter le chapitre 40 en priorité et d'avoir des conventions obligatoires pour l'introduction du chapitre 40 parce que le Conseil fédéral veut une décision définitive quant au 


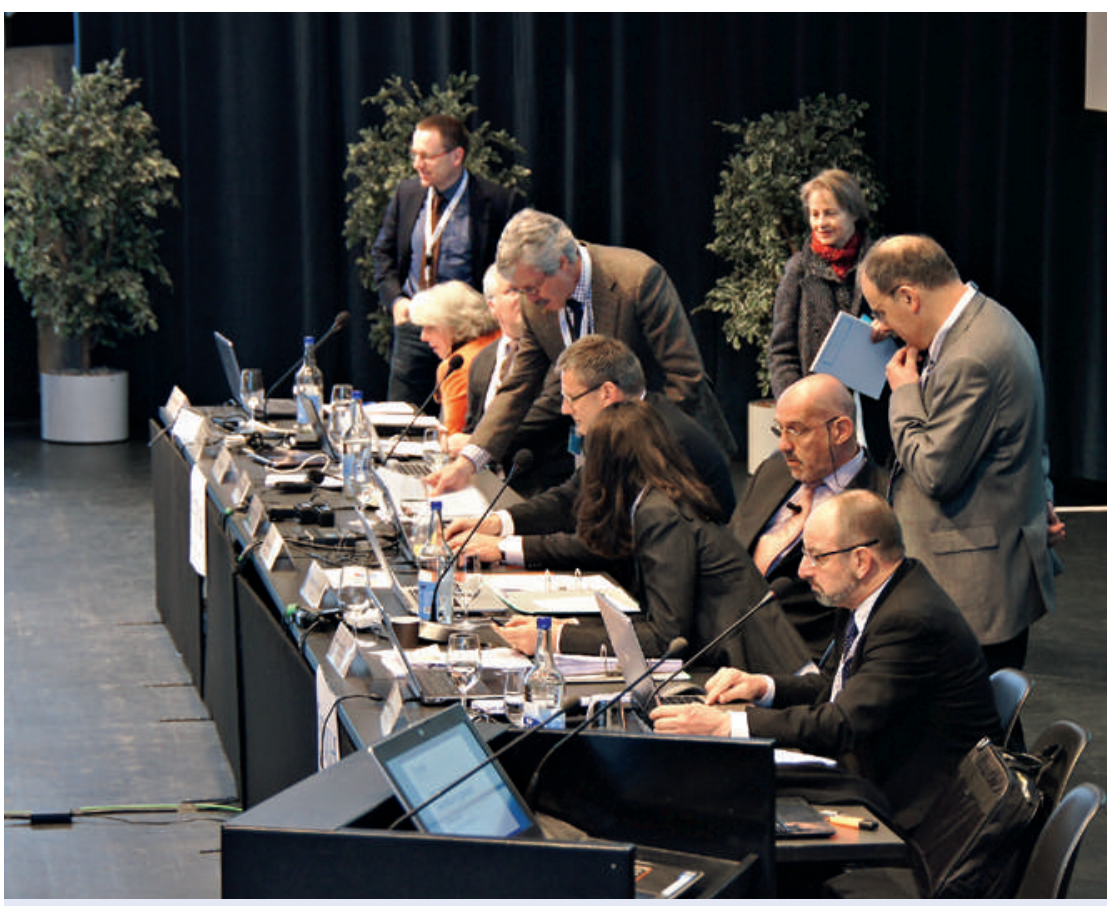

que celle qui règne sur le podium. puissent y renoncer. La FMH déclare clairement vouloir la mise en œuvre de TransAL en été 2013, comme indiqué dans le Masterplan, mais avec une représentation correcte du laboratoire au cabinet médical. La FMH a élaboré une liste qui comprend 45 analyses à facturer selon le tarif «Point of Care». Des discussions intensives sont en cours avec l'OFSP.

\section{Remise de médicaments par les médecins}

La collaboration entre la FMH, H+ et la CTM se déroule de manière efficace et constructive au niveau technique. santésuisse prétend que les marges actuelles sont trop élevées, que les prestations médicales sont rémunérées dans le TARMED par la liste des analyses et que les frais de logistique sont indemnisés dans le modèle de coûts GRAT du TARMED. Mais la FMH dispose des données, des chiffres, de l'expertise et du savoir-faire qui lui ont permis de réfuter clairement les allégations de santésuisse. La FMH a exigé une rectification écrite à ce sujet, qu'elle a obtenue. Ce succès partiel donne du courage et la FMH poursuivra les négociations de manière constructive et innovatrice pour des tarifs justes et adéquats.

retrait de l'initiative «Oui à la médecine de famille» d'ici au printemps 2013.

Liste des analyses «Trans $A L »$ :

la FMH demande une rémunération correcte

La situation pour les laboratoires au cabinet médical n'est pas du tout satisfaisante. Depuis l'introduction de la liste révisée des analyses en été 2009, le chiffre d'affaires des laboratoires au cabinet a baissé de 18 à $30 \%$ selon les spécialisations. Ainsi que la FMH le demandait depuis longtemps, l'OFSP a lancé un projet pour la révision de la liste des analyses (TransAL) dans le cadre du «Masterplan Médecine de famille». Celle-ci prévoit d'introduire d'ici à l'été prochain un chapitre spécial pour les «analyses rapides» au laboratoire du cabinet médical. Un groupe d'experts mis sur pied par l'OFSP a défini dix-huit analyses que le cabinet médical peut facturer dans le tarif «Point of Care». Toutes les autres analyses effectuées au laboratoire du cabinet médical doivent être facturées aux normes des laboratoires mandatés, ce qui correspond à une perte de 50 à $60 \%$ par analyse selon les calculs de la FMH et donc à la disparition du laboratoire au cabinet médical. Le groupe d'experts de l'OFSP, qui n'a pas de médecin en pratique privée dans ses rangs, pense que l'amélioration du tarif des analyses réalisées au laboratoire du cabinet médical créerait de faux incitatifs. Pourtant, les calculs de la FMH montrent que l'introduction de la liste révisée des analyses a fait chuter le chiffre d'affaires du laboratoire au cabinet médical, alors que le nombre des analyses effectuées n'a presque pas diminué. Il s'avère que le laboratoire représente un instrument de travail bien trop important pour que les médecins

\subsubsection{Domaine Données, démographie et qualité} (DDQ) - (information succincte)

Christoph Bosshard/CC informe de la réussite de la création de l'«Académie suisse pour la qualité en médecine (ASQM)» fin novembre 2012. Avec l'ASQM, la FMH agit au niveau national en faveur des patients et souligne sa volonté de jouer un rôle d'interlocuteur central pour tous les thèmes relatifs à la qualité médicale dans le domaine de la santé. Avec son champ d'activité étendu, l'ASQM est vouée à devenir un forum d'échange interdisciplinaire au sein du corps médical, auquel seront associés différents partenaires du système de santé. Pour l'instant, il s'agit d'animer cet organe nouvellement créé. Ch. Bosshard invite les sociétés de discipline médicale, les sociétés cantonales de médecine, les organisations faîtières, l'ASMAC et l'AMDHS à annoncer leurs délégués d'ici au $1^{\text {er }}$ mars 2013 au plus tard. Il rappelle les cinq points formulés par l'ASQM à l'égard du corps médical: participer, proposer, discuter, donner son avis et soutenir. Dans cet esprit, Ch. Bosshard invite le corps médical à faire connaître ses attentes et à participer à l'enquête qui sera réalisée dans le but de dresser l'inventaire des instruments concernant la qualité, et ainsi à contribuer au travail de l'ASQM et à le soutenir.

\subsection{3 eHealth - infrastructure de sécurité}

Urs Stoffel/CC décrit la situation actuelle autour de la carte de professionnel de santé (HPC). La HPC est plus qu'une simple carte, c'est une carte de médecin électronique qui peut être dotée de différents certificats, tel un trousseau de clefs, permettant de communiquer avec d'autres systèmes informatiques. La 
FMH a toujours été claire et fait savoir qu'elle tenait à éditer la HPC. Par ailleurs, c'est à elle qu'il incombe de décider qui est médecin. Ajoutons que la HPC gagne en importance pour deux raisons. D'une part, le projet relatif au dossier électronique du patient (loi DEP) arrivera probablement au Parlement durant la session d'été 2013. D'autre part, le nouveau droit de la protection de l'adulte entrera en vigueur le $1^{\text {er }}$ janvier 2013. Celui-ci prévoit que les directives anticipées du patient pourront également être enregistrées sur la carte d'assuré. La HPC doit donc pouvoir communiquer avec cette carte. Mais actuellement, deux systèmes cohabitent, ce qui complique la situation. La carte d'assuré de la Helsana est munie d'un certificat compatible avec la HPC de la FMH alors que la carte d'assuré délivrée par santésuisse n'est pas en mesure de communiquer avec la HPC. Des discussions sont en cours avec les assureurs afin de trouver une solution.

La carte d'assuré contient les données administratives obligatoires pour le décompte des prestations LAMal. Les données personnelles sont facultatives. Pour ces dernières, la FMH estime que la sécurité des patients n'est pas assurée. En effet, cette carte ne contient qu'une part infime des données thérapeutiques significatives; elle n'offre pas non plus la garantie que ces données sont à jour et complètes; en outre, aucun back-up n'existe en cas de perte de la carte sur laquelle ne figure aucune photo. Si elle est attribuée à une personne autre que son destinataire, elle peut même devenir dangereuse en cas d'urgence en raison de données erronées.

$U$. Stoffel relève que le processus n'est pas encore bien réfléchi. La FMH pense que des données d'urgence compilées sur la carte ne peuvent en aucun cas être l'objectif poursuivi ni une solution d'avenir. Au contraire, il s'agit de poursuivre le développement optimal du dossier électronique du patient. Pour la FMH, le projet du Conseil fédéral n'est pas encore mûr et la FMH a tout intérêt à participer de manière constructive à la recherche de solutions.

\subsection{ISFM}

Plate-forme «Avenir de la formation médicale» Werner Bauer, président de l'ISFM, informe la Chambre médicale des résultats de la plateforme «Avenir de la formation médicale», mise en place par l'OFSP et la CDS. Les travaux du premier groupe «Financement de la formation postgrade des médecins» sont achevés. Le deuxième groupe «Harmonisation des formations universitaire, postgrade et continue des médecins» vient de rédiger un rapport final. Compte tenu de l'intention du Conseil fédéral de créer au moins 300 places d'étude supplémentaires en médecine humaine, ce rapport préconise une régulation de la formation postgraduée des spécialistes. Or cette régulation visant à former le «nombre requis» de chirurgiens plastiques, dermato- logues, médecins de famille et psychiatres est extrêmement complexe. Elle constitue un sujet de discussion dans de nombreux pays, sans grand succès pour l'instant. L'OFSP se penche également sur la question en créant un nouveau groupe de travail auquel l'ISFM participera. Et enfin, le troisième groupe de travail «Interprofessionnalité» veille à institutionnaliser la collaboration interprofessionnelle et à mieux coordonner les cursus de formation des professions de la santé.

\section{Atelier «Teach the Teachers»}

En collaboration avec le «Royal College of Physicians of London», l'ISFM a organisé trois ateliers portant sur l'«Achieving excellence as a medical educator». Organisés à Lucerne en septembre 2012, sous forme de projet-pilote, ces ateliers ont eu pour but de sensibiliser les jeunes médecins-chefs à leur nouveau rôle d'enseignant et de supérieur hiérarchique en se concentrant sur des questions clés telles que l'enseignement, l'évaluation, le feed-back et le leadership. Ces ateliers ont connu un énorme succès, toutes les places disponibles ont été réservées en l'espace de deux jours. Pour répondre à l'énorme demande, ils seront à nouveau organisés en avril et en septembre 2013.

\section{Systématique des titres}

L'ISFM a été sollicité de plusieurs parts pour élargir son système de titres de manière à ce que les spécialisations soient mieux prises en compte. L'ISFM a donc décidé d'accorder par principe la possibilité d'acquérir aussi une formation approfondie dans le cadre de la formation postgraduée régulière d'une discipline. L'art. 12 al. 2 de la Réglementation pour la formation postgraduée (RFP) a été révisé en conséquence: «Un titre de spécialiste peut comprendre une ou plusieurs formations approfondies représentant des spécialisations / approfondissements au sein d'une même discipline. Les formations approfondies peuvent être acquises au cours de la formation postgraduée pour l'obtention du titre de spécialiste ou au moyen d'une formation postgraduée supplémentaire. (...)». C'est de la compétence des sociétés de discipline médicale de savoir si des formations approfondies doivent être définies, et le cas échéant lesquelles. Voici un exemple: le docteur Hans Muster, spécialiste en médecine interne générale FMH, spécialisation: médecine de famille; ou la doctoresse Ruth Muster, spécialiste en neurochirurgie FMH, spécialisation: chirurgie de la colonne vertébrale.

Le président ouvre la discussion. Dans le contexte des places d'étude supplémentaires, Vincenzo Liguori/ TI mentionne la faculté de médecine prévue au Tessin et demande au corps médical de soutenir ce projet. W. Bauer confirme l'élan de sympathie en faveur de ce projet, malgré les nombreuses difficultés organisationnelles et financières et il l'assure de son soutien. 


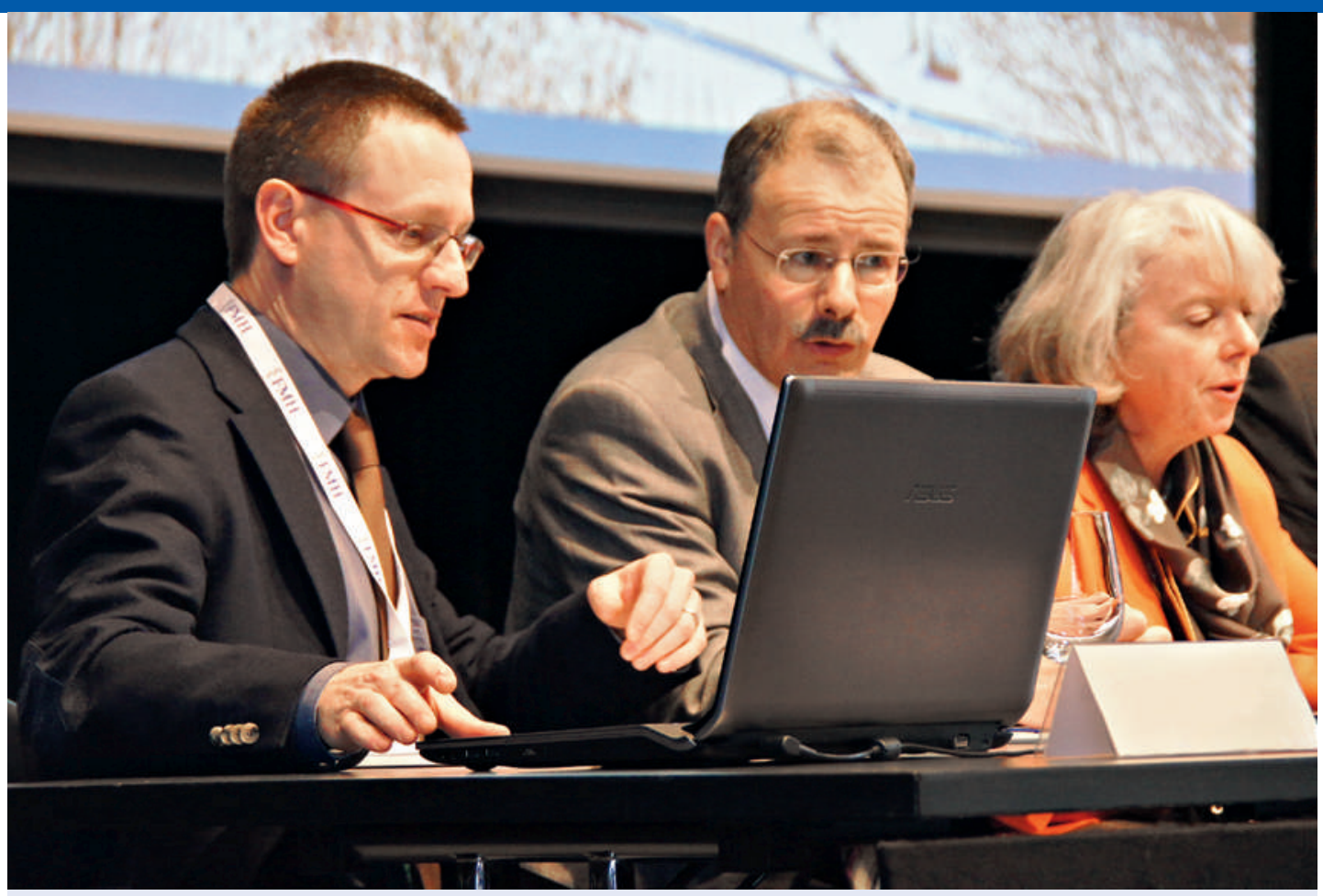

Jürg Schlup (au centre), président de la FMH depuis le 7 décembre 2012, Christoph Bosshard et Monique Gauthey suivent avec attention les débats concernant les différents points à l'ordre du jour.

\subsection{Secrétariat général}

La secrétaire générale Anne-Geneviève Bütikofer fait le point sur la réorganisation du Secrétariat général de la FMH. Suite à l'analyse stratégique de la structure opérationnelle effectuée en automne 2011, les premières mesures ont pu être déployées dès le début de l'année. D'ici à la fin 2012, le Secrétariat général sera doté d'une nouvelle structure avec notamment un nouveau Secrétariat central et un Conseil de direction regroupant la secrétaire générale et ses deux suppléants. Une plus grande importance est attribuée aux ressources humaines avec, entre autres, la création d'un service $\mathrm{RH}$ et l'introduction d'une nouvelle réglementation du temps de travail et des heures supplémentaires. La réorganisation se concentre actuellement sur l'examen et l'optimisation des règlements, des procédures et des processus internes ainsi que sur le développement de la politique du personnel. Une attention particulière est accordée à la gestion financière: mise en pratique du nouveau processus de planification et de budgétisation, introduction d'un outil de pilotage pour mieux gérer les projets et les coûts, amélioration des chiffres clés pour le budget et des rapports trimestriels et semestriels. Un «forecast» sera en outre mis en place. Lors d'une prochaine étape, la réorganisation portera sur l'extension du système de contrôle interne et l'introduction d'un contrôle des risques. Enfin, il s'agira de se pencher sur l'introduction d'un enregistrement centralisé des documents et sur la mise à jour des procédures d'archivage. Parallèlement, le Secrétariat général procédera à la révision de la procédure de gestion et de suivi des aides financières et du sponsoring, et actualisera la stratégie de communication interne et externe. Il s'agira également d'examiner les prestations aux membres et la mutualisation des collaborations avec les sociétés cantonales et sociétés de discipline, mais aussi avec d'autres partenaires. Et enfin, nous devrons également reconsidérer les locaux qui vieillissent et deviennent trop exigus. A.-G. Bütikofer souligne que la réorganisation du Secrétariat général prendra encore du temps et qu'elle accompagnera la FMH également ces prochains mois voire même ces prochaines années. Tout cela dans le but d'améliorer les prestations de services pour les membres de la FMH - dans le respect du maintien des dépenses - et de mieux soutenir les membres du Comité central.

\section{Abus à caractère sexuel lors de l'activité médicale}

\subsection{Modification du Code de déontologie et du Règlement de la Commission de déontologie}

\subsection{Modification de l'art. 49 du \\ Code de déontologie}

\subsection{Abus à caractère sexuel lors de} l'activité médicale

Le président donne la parole à Christine Romann/CC qui dirige le groupe de travail «Abus à caractère sexuel lors de l'activité médicale» et défend la proposition $n^{\circ} 1$ au point $n^{\circ} 3.1$ du Comité central et de l'Assemblée des délégués. C. Romann explique qu'il devient urgent de procéder à des changements car les procédures manquent entièrement de transparence pour les patient-e-s. En règle générale, cela se 
termine toujours par la parole d'une des parties contre celle de l'autre, mais les patient-e-s ignorent quelle est la position du médecin, et ils ne sont pas non plus associés à la procédure. C. Romann ajoute que l'objectif visé est la disparition de tout abus à caractère sexuel lors de l'activité médicale. Mais il est évident qu'il s'agit là d'un objectif idéal que nous ne pourrons jamais atteindre complètement. Nos efforts devraient toutefois viser ce but. A cet effet, le groupe de travail propose deux voies: d'une part, ancrer cette question dans la formation postgraduée et continue de manière à susciter un effet préventif; d'autre part, réagir de manière adéquate lorsqu'un abus à caractère sexuel s'est produit. C. Romann précise que c'est à ces médecins, hommes ou parfois aussi femmes, à qui cela arrive une fois, dans un moment de crise existentielle, dans une situation donnée, que nous aimerions offrir notre soutien, par exemple avec des directives similaires à celles de la Société suisse de gynécologie et d'obstétrique comme aussi par la structure ReMed. Dans les cas de récidive, il est indispensable que les patient-e-s dénoncent les faits, sinon les organisations médicales ne peuvent prendre aucune mesure contre les coupables. C. Romann estime que l'une des raisons de ne pas porter plainte réside dans la procédure ellemême. Après avoir porté plainte, les patient-e-s concerné-e-s ne reçoivent plus aucune information pendant des mois, jusqu'à la prise d'une décision; c'est ce qui est décourageant et qui doit être changé, d'où la proposition $\mathrm{n}^{\circ} 3.1$ pour la modification de l'article 45 du Code de déontologie de la FMH qui stipule actuellement que la personne qui porte plainte doit être membre de la FMH pour pouvoir bénéficier du statut de partie lors de la procédure. Désormais, les patient-e-s doivent également avoir la possibilité d'avoir la qualité de partie.

Article 45 alinéa 2 (nouveau): Ont qualité pour être partie les personnes suivantes qui ont un intérêt personnel légitime au résultat de la procédure et qui en font la déclaration par écrit à la commission de déontologie de première instance: $a$. membres de la FMH; $b$. patientes et patients lors de procédures pour cause d'abus de l'état de dépendance sur le plan sexuel.

Une modification de l'article 4 du Code de déontologie est également nécessaire dans sa version allemande; la version française n'appelle aucun changement:

Article 4: (...)

Dans l'exercice de sa profession, le médecin n'exploite pas l'état de dépendance du patient; il lui est tout particulièrement interdit d'abuser de son autorité sur lui, tant sur le plan émotionnel ou sexuel que matériel.

Ch. Romann est convaincue que la relation médecin-patient est toujours une relation de dépendance. La proposition du Comité central et de l'Assemblée des délégués contient en outre une modification des articles 9, 17 et 22 du Règlement de la Commission de déontologie. Elle vise une meilleure collaboration avec les autorités de surveillance, et notamment à accorder la qualité de partie aux patient-e-s lors de procédures contre des abus à caractère sexuel dans le cadre d'une relation de dépendance.

Beat Gafner/BE dépose une motion d'ordre afin que la Chambre médicale vote sur l'entrée en matière avant de s'engager dans la discussion de la proposition.

- Cette proposition est rejetée par 97 voix contre 39 et deux abstentions.

Ch. Romann passe ensuite à la proposition $\mathrm{n}^{\circ} 1 \mathrm{au}$ point $\mathrm{n}^{\circ} 3.2$ de l'Association des médecins du canton de Genève, présentée par Pierre-Alain Schneider, et demandant une modification de l'article 49 Procédure officielle en cours du Code de déontologie de la FMH: Article 49 (actuel): Si, pour la même affaire, une procédure officielle est engagée par une autorité administrative ou un tribunal, la procédure interne peut être suspendue, voire annulée.

Article 49 (complété): Néanmoins, lorsque la plainte porte sur une violation du respect de la dignité humaine ou un abus de l'état de dépendance du patient, la commission procédera à une première audition dans les meilleurs délais.

Ch. Romann salue la proposition de l'Association des médecins du canton de Genève. L'expérience montre que les procédures sont presque toujours suspendues. Cet article contribue à ce qu'elles ne puissent plus l'être lorsqu'il s'agit d'un abus à caractère sexuel.

J. de Haller donne la parole à Pierre-Alain Schnei$\mathrm{der} / G E$ qui voit sa proposition comme un signe favorable pour les patient-e-s dont la dignité humaine a été lésée, mais aussi comme un signal vers l'extérieur. Les Commissions de déontologie doivent traiter avec la plus stricte priorité tous les abus commis dans le cadre d'une relation de dépendance et c'est à ce titre que cela doit figurer dans le Code de déontologie.

- La proposition $n^{\circ} 1$ au point $n^{\circ} 3.2$ est acceptée à une majorité évidente, avec deux voix contre et cinq abstentions.

Ch. Romann/CC présente la proposition $\mathrm{n}^{\circ} 1$ au point $\mathrm{n}^{\circ} 3.3 \mathrm{du}$ VEDAG, de la SMSR et de l'OMCT, représentés par Peter Wiedersheim/SG. Les organisations faîtières des sociétés cantonales de médecine demandent de rejeter les modifications du Code de déontologie de la FMH (articles 4 et 45) afin de pouvoir encore approfondir la question, y intégrer les éléments positifs des règlementations en partie déjà existantes dans les cantons et les sociétés de discipline médicale, et pour élaborer des mesures préventives d'accompagnement. C. Romann relève qu'un excellent travail a été réalisé dans quelques cantons et que d'autres accusent encore un certain retard. Mais la proposition du Comité central et de l'Assem- 
blée des délégués poursuit un autre objectif, à savoir améliorer la procédure. Pour cela, il n'est pas nécessaire de consulter les cantons. La collaboration avec les cantons aura lieu plus tard sous la forme d'une offre. Chaque canton peut, par exemple, décider luimême de la création d'une instance de médiation.

Le président donne la parole à Peter Wiedersheim/ SG pour défendre la proposition de la Société cantonale de médecine de St-Gall. $P$. Wiedersheim/SG souligne qu'il ne s'agit pas de nier le besoin d'agir dans le cas des abus à caractère sexuel. Il veut rejeter les propositions de modification pour les améliorer et saisir l'occasion de régler cette affaire délicate et importante de la meilleure manière possible. Il estime que les bonnes réglementations des sociétés cantonales de médecine, mais aussi des sociétés de discipline médicale, n'ont pas été prises en compte de manière systématique. La formulation concernant la relation de dépendance ne correspond pas à l'image d'un patient majeur. Chaque médecin est sensibilisé au cours de sa formation à prendre garde à ce qu'aucune relation de dépendance ne s'instaure avec le patient. En outre, Peter Wiedersheim critique l'absence de mesures d'accompagnement et de propositions concrètes pour les patient-e-s et les médecins concernés ainsi que l'aspect de la détection précoce. Il recommande, au nom des trois organisations faîtières VEDAG, SMSR et OMCT, de rejeter la proposition du Comité central et de l'Assemblée des délégués. Il se déclare prêt à collaborer pour que le Comité central puisse présenter à la prochaine Chambre médicale avec confiance une solution susceptible d'obtenir la majorité des voix.

Le président ouvre la discussion. Pour Véronique Monnier-Cornuz/VD, les sociétés cantonales sont les mieux placées pour traiter le problème délicat que posent les abus à caractère sexuel. Il n'y a aucune raison d'interférer dans l'autonomie des sociétés cantonales. Hanspeter Kuhn/FMH donne raison à l'oratrice précédente quand elle dit qu'un excellent travail est effectué dans un grand nombre de cantons. Mais la question qui se pose ici est une autre. Les droits des parties ne peuvent être réglés qu'au niveau national avec le Code de déontologie. Les cantons ne peuvent rien modifier d'eux-mêmes.

Philippe Vuillemin/VD estime que la présentation de Christine Romann est justifiée. Néanmoins, il se heurte à des formulations comme «la patiente» et «le médecin» qui ne sont pas sexuellement neutres. C. Romann répond qu'elle accepte volontiers cette remarque, qu'elle y tient beaucoup et qu'elle s'efforce déjà maintenant d'utiliser une formulation neutre dans tous les documents. Ph. Vuillemin est satisfait de sa réponse.

Daniel Schröpfer/ASMAC revient à la proposition proprement dite du Comité central et de l'Assemblée des délégués. L'ASMAC l'approuvera parce qu'elle permet de renforcer les droits des patientes et des patients. Certains aspects doivent toutefois être encore revus. Hans Ulrich Iselin/AG estime que même si la proposition du VEDAG, de la SMSR et de l'OMCT est approuvée, il faudrait voter, dans le texte allemand, sur l'expression «das Abhängigkeitsverhältnis» ou «ein Abhängigkeitsverhältnis» afin de clarifier ce point important.

Le président passe aux votes: le premier concerne la question de savoir si la Chambre médicale préfère la formulation «das Abhängigkeitsverhältnis» ou «ein Abhängigkeitsverhältnis». Elle vote à une large majorité en faveur de la formulation «ein Abhängigkeitsverhältnis»*. Le deuxième vote concerne la proposition de renvoi présentée par le VEDAG, la SMSR et l'OMCT.
* La version française stipule actuellement «Dans l'exercice de sa profession, le médecin n'exploite pas l'état de dépendance du patient; ...»

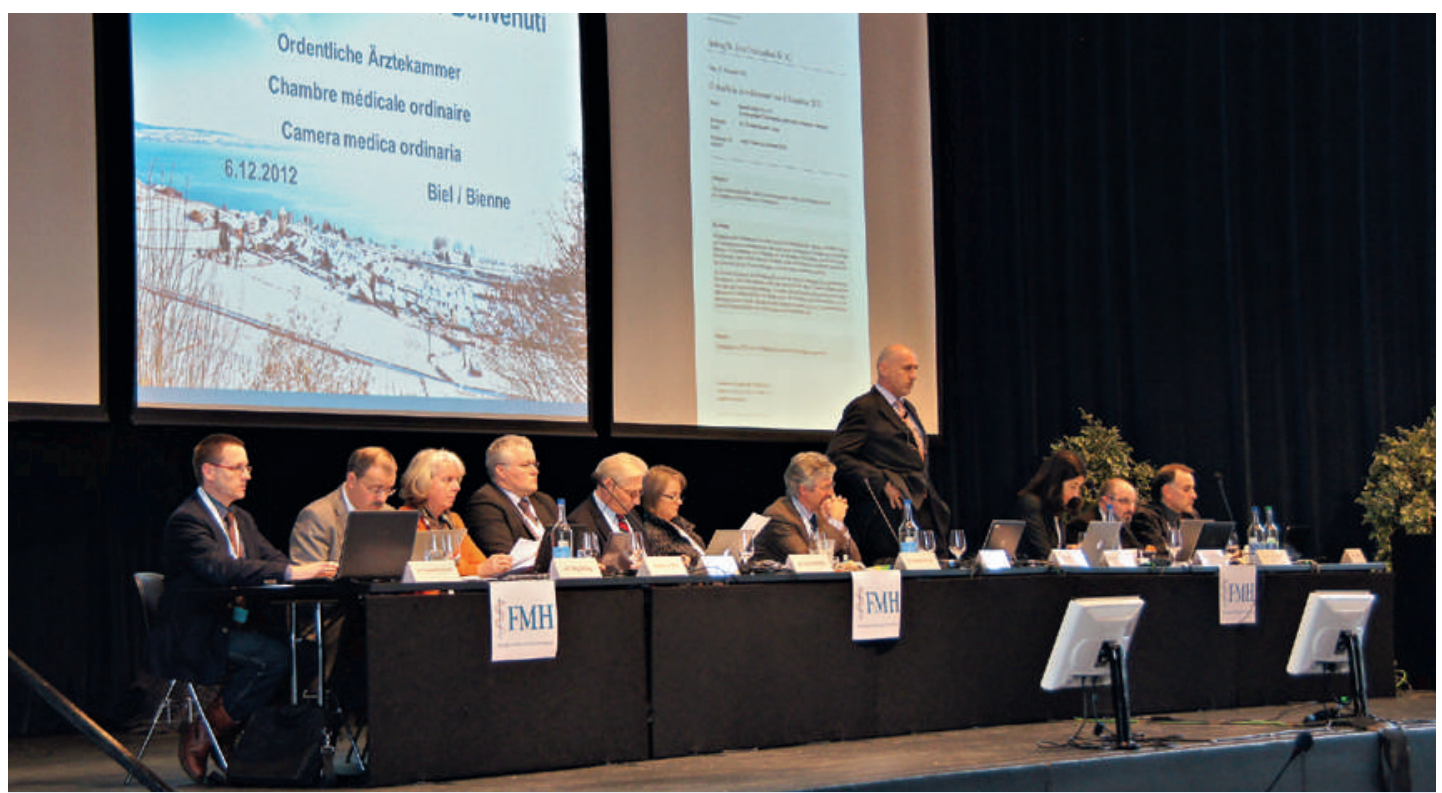

Le Comité central orchestre le déroulement de la Chambre médicale. 
- Le vote sur la proposition $n^{\circ} 1$ au point 3.3 se solde par un ex aequo: 69 oui contre 69 non et deux abstentions.

C. Romann avoue qu'elle ne serait pas à l'aise si le résultat d'un éventuel nouveau vote ne devait dépendre que d'une seule voix. La discussion a montré que l'heure n'était pas encore mûre. Elle retire donc la proposition au nom du Comité central, en remerciant vivement les délégués d'avoir mené cette discussion importante. Elle est tout à fait disposée à présenter une nouvelle mouture de cette proposition à la Chambre médicale du printemps prochain.

Jacques de Haller estime aussi qu'il s'agit d'une décision sage. Cela permettra de revoir certains points puis de présenter le projet une troisième fois à la Chambre médicale.

\section{Allocution du nouveau président}

Peu avant la pause de midi, Jürg Schlup, le nouveau président de la FMH, prend la parole: «Dès demain matin, je reprendrai la présidence de la FMH. Je m'en réjouis et je m'efforcerai de représenter le mieux possible vos intérêts. Ce ne sera pas toujours possible, vous le savez aussi bien que moi. Je chercherai le dialogue avec vous parce que je suis convaincu que les conflits doivent être mis sur la table et discutés. Je veux que l'on puisse parler de nos désaccords pour que nous parvenions à un véritable consensus, pour que nous unissions nos forces et pour que nous parlions d'une seule voix vers l'extérieur. Nous ne devons pas toujours être du même avis, mais nous devrons toujours parler d'une seule voix. Je souhaite venir à votre rencontre. Ces dernières semaines, j'ai déjà effectué quelques visites - et j'en ferai d'autres encore - auprès de la Société de médecine du canton de Soleure, de l'Association des Médecins du Canton de Genève, de l'Ordine dei medici del Cantone Ticino, de la fmCh, des chirurgiens, des médecins de famille et de l'ASMAC. Je me réjouis de recevoir vos invitations afin de pouvoir entretenir le dialogue avec vous. Je m'engagerai pour que vous conserviez votre liberté thérapeutique. Je m'engagerai pour que vous puissiez exercer votre profession dans de bonnes conditions. Je m'engagerai pour une rémunération adéquate de notre profession et pour des soins médicaux de qualité élevée, dans un système accessible à tous qui profite aux patients. Il faut voir le changement comme une chance et je vais m'employer à en faire usage dans votre sens. Je vous remercie d'ores et déjà du soutien que vous m'apporterez.»

\section{Allocution du conseiller fédéral Alain Berset}

Après la pause de midi, la Chambre médicale a le plaisir de recevoir le conseiller fédéral Alain Berset. A l'instar de la FMH, le chef du DFI place les patientes et les patients au centre de sa politique en matière de santé. Le conseiller fédéral estime que préserver la qualité de vie et la qualité des soins, renforcer l'égalité des chances et améliorer la régulation de la prise en charge médicale seront les grands défis de demain et qu'il est possible d'en venir à bout malgré la complexité du système de santé helvétique. Pour les relever, continue Alain Berset, tous les acteurs «doivent agir de manière consensuelle et intervenir pour trouver en commun des solutions équilibrées». Le conseiller fédéral appelle les délégués de la FMH à collaborer au développement d'un système de santé durable et à soutenir sa modernisation, notamment en développant de nouveaux modèles de soins ou le dossier électronique du patient.

\section{Prise de congé de Jacques de Haller}

Ernst Gähler prend la parole pour remercier Jacques de Haller de son engagement et des services qu'il a rendus au corps médical et à la FMH. Même s'il est bien évident qu'ils n'ont pas toujours été du même avis, il faut noter que des solutions ont toujours été trouvées pour le corps médical et les patients. Durant les années passées à la tête de la FMH, Jacques de Haller est devenu un président à l'écoute, prêt à modifier son opinion quand les faits le justifient. De plus, Jacques de Haller a posé des jalons au niveau international où il a également su se faire entendre. «Lorsque je pense à ces six dernières années, je vois tout le chemin parcouru. Je t'ai connu comme un président très réactif, capable de discuter des divergences et de rechercher des solutions communes. Avec la FMH, tu as atteint bien des objectifs et tu as effectué un travail considérable. Pour cela aussi, j'aimerais vivement te remercier. Je te souhaite le meilleur pour l'avenir, ainsi qu'une excellente santé et te dis: tout de bon.»

Pierre-François Cuénoud s'adresse également au président sortant. Il rappelle d'abord son élection en 2004. Une tâche herculéenne attendait Jacques de Haller. Après avoir découvert bon nombre de montages financiers complexes et opaques, sa première action a été de permettre à la FMH de tirer ceux-ci au clair et de s'en désengager. Jacques de Haller a introduit un style de conduite très participatif, donnant la parole à chacun, faisant sienne la maxime de Voltaire: «Je ne suis pas d'accord avec ce que vous dites mais je me battrai toujours pour que vous puissiez le dire.» Il en a résulté une culture du débat permettant au Comité central de mûrir ses décisions au cours des séances. Enfin, son respect des différences, cette faculté de les considérer plus comme une richesse que comme un handicap, a permis aux spécialistes et aux médecins hospitaliers de se sentir respectés, d'avoir le sentiment d'appartenir à une fédération où ils ont aussi leur place. Après d'âpres négociations internes, Jacques de Haller a aussi obtenu la création de l'Assemblée des délégués. P.-F. Cuénoud souligne que la FMH a gagné en efficacité à l'interne et en poids politique à l'externe.

P.-F. Cuénoud poursuit avec quelques anecdotes qui restent de ces huit ans passés. Le jeu de mains de Jacques de Haller restera dans les mémoires des membres du Comité central, frappant quatre fois 
l'une contre l'autre, non pour applaudir les performances mais pour signifier la reprise du travail après une pause systématiquement plus longue que prévue. Ces mains de joueur de karaté ont aussi occasionné quelques maladresses. La table en verre dans le bureau de la Secrétaire générale en fut deux fois victime, d'abord à son arrivée puis à son départ lors d'une reconstitution du premier épisode. P.-F. Cuénoud relate aussi quelques «performances sportives» qui vont également rester dans les annales, particulièrement celles d'une ascension mémorable en chaussures de ville lors d'une sortie du personnel dans le Haut Valais avec tout le harnachement d'une technologie de l'information adéquate. «Il faut dire que le laptop ou la tablette Mac ne te quitte jamais. Toi, le Lucky-Luke du courrier électronique qui répond plus vite qu'un clic de souris, que ce soit au milieu d'une séance ou à une heure quarante-trois du matin.»

Ensuite, P.-F. Cuénoud se rappelle un dîner officiel à l'Assemblée européenne des médecins dirigeants d'hôpitaux, lors duquel l'allocution de Jacques de Haller, dans un anglais parfait, a laissé libre cours à la dimension internationale du personnage. Les esprits ont été marqués et nombreux sont ceux qui lui ont dit: «Vous avez de la chance d'avoir un Président de cette trempe.»

P.-F. Cuénoud qualifie le bilan des deux législatures sous la présidence de Jacques de Haller de très positif. «Tu as su fédérer les forces pour parvenir à des résultats tangibles, tu as fait de la FMH un partenaire essentiel sur l'échiquier politique.» Certes, son caractère fort et ses convictions fortes ont parfois dérangé mais elles n'ont jamais empêché son respect démocratique et indéfectible de l'opinion majoritaire. P.-F. Cuénoud remercie Jacques de Haller pour ces belles années et lui souhaite une nouvelle activité à la mesure de ses capacités.

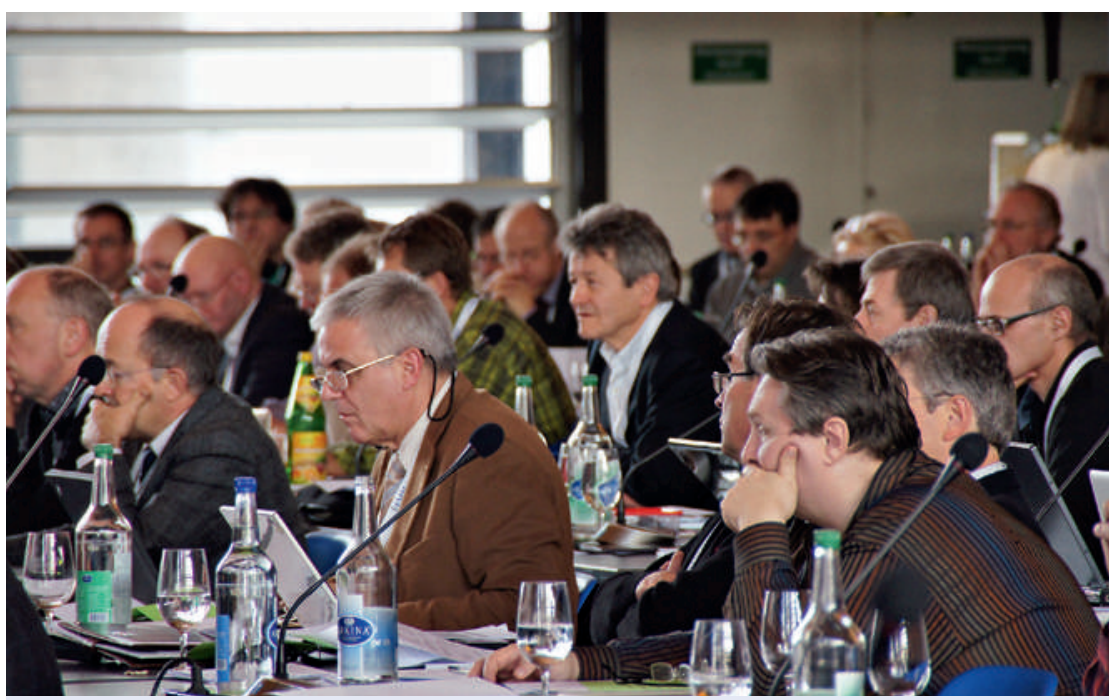

Entre deux débats passionnés, la Chambre médicale laisse aussi place à la réflexion.

\section{Allocution de Jacques de Haller}

Dans son allocution, le président Jacques de Haller évoque le chemin parcouru, la volonté d'avoir une FMH forte, moderne et démocratique, ce qui a conduit la FMH à être aujourd'hui une des organisations parmi les plus influentes. Mais ce développement s'accompagne de grandes responsabilités, qu'il faut assumer correctement. Cela veut dire que l'on sait où l'on veut aller, et surtout que l'on sait pourquoi; que l'on connaît ses objectifs, mais surtout qu'on les enracine dans une vision qui les précède, qui les dépasse, et qui les valide. Pendant ses huit ans et demi de présidence, J. de Haller a eu comme vision une médecine qui s'engage passionnément pour les patients, dont le bien-être ne se résume pas à être libéré de leurs douleurs. Bien plus, des patients qui vont bien, ce sont des gens qui sont aptes et libres d'épanouir leur créativité, de la vivre dans la société et de pouvoir être eux-mêmes. A la FMH, J. de Haller a accordé une grande importance à la liberté de parole. Dans cet esprit, il invite les membres de la FMH à ne pas se laisser tenter par l'intolérance, à accepter les divergences, à ne pas avoir peur d'instances fortes et à faire preuve de confiance envers leurs dirigeant-e-s. Par ailleurs, le président recommande à la FMH de vivre en lien avec la société, de ne pas s'exclure de ses évolutions et de réfléchir constamment à la spécificité du médecin. Il ajoute que sans une éthique rigoureuse, les médecins ne pourront prétendre garder la confiance que la population place en eux. J. de Haller termine son allocution en précisant qu'il ne sait pas encore où son chemin professionnel le mènera. Mais la FMH peut compter sur sa loyauté. Le texte complet de l'allocution sera publié dans un prochain numéro du Bulletin des médecins suisses.

\section{Budget 2013 de l'ISFM, de la CdG et de la FMH}

Jacques de Haller donne la parole à Roland Schwarz de la Commission de gestion (CdG).

Roland Schwarz/CdG s'exprime rapidement sur deux points. Le premier concerne la liste des salaires de 2013 des membres du Comité central. Celle-ci a été remise à tous les délégués à la Chambre médicale ayant droit de vote. Mais $R$. Schwarz précise que cette liste ne correspond pas à la décision de la Chambre médicale du 10 décembre 2009 qui stipule que «le revenu annuel des membres du Comité central est communiqué chaque année par écrit aux délégués à la Chambre médicale». Cette liste est donc établie après coup. En revanche, la liste distribuée aujourd'hui indique des salaires bruts probables. Par ailleurs, elle est incomplète. $R$. Schwarz demande au Comité central de remettre aux délégués ayant droit de vote, lors de la prochaine Chambre médicale d'avril 2013, une liste conforme à la décision de la Chambre médicale.

Le second point abordé par $R$. Schwarz concerne l'indemnité de départ du président. L'information 
relayée par certains médias selon laquelle il existerait une réglementation spéciale pour le président sortant est inexacte. Il n'y a jamais eu de telle réglementation. La réglementation en vigueur au moment de l'entrée de fonction de Jacques de Haller a été modifiée en 2010 par la Commission de gestion. De ce fait, l'ancienne réglementation est encore valable pour le président sortant et elle est parfaitement légale. Elle n'aurait pu être modifiée pour Jacques de Haller que par une résiliation de contrat pour cause de modification. Rappelons que la nouvelle réglementation n'est plus aussi généreuse.

$J$. de Haller remercie de ces précisions et il donne la parole à Christoph Hänggeli/ISFM pour présenter le budget de l'ISFM (proposition $n^{\circ} 1$ au point $n^{\circ} 4.1$ du comité de l'ISFM).

\subsection{Budget 2013 de l'ISFM}

Christoph Hänggeli/ISFM explique que l'année prochaine, il ne faudra pas s'attendre à de grands changements concernant le budget de l'ISFM. Les recettes principales proviennent des taxes encaissées pour la remise de titres. Une certaine consolidation s'est produite ces dernières années. L'ISFM a de nouveau délivré un nombre de titres légèrement supérieur à la moyenne et il prévoit également une légère augmentation pour 2012. Ch. Hänggeli souligne que près d'un tiers de tous les diplômes fédéraux sont remis à des médecins étrangers. Il ne connaît aucun pays doté d'un chiffre aussi élevé. Les titres FMH semblent donc être attrayants. On a l'impression que tous les médecins étrangers accomplissant leur formation postgraduée dans notre pays veulent également acquérir leur titre de spécialiste en Suisse, alors qu'ils pourraient probablement l'acquérir plus vite et bien meilleur marché dans leur pays d'origine.

Ch. Hänggeli s'attend pour 2013 à des recettes légèrement supérieures à celles de 2011. Elles permettront à nouveau à l'ISFM de présenter un budget plus ou moins équilibré avec un déficit modéré de CHF 125000.-. Ch. Hänggeli souligne qu'il n'y a pas lieu d'augmenter les taxes. Au contraire, si le bouclement des comptes est encore meilleur que les prévisions, on pourra même les baisser l'année prochaine.

$J$. de Haller prend acte qu'aucune question n'est posée concernant le budget de l'ISFM et il invite Roland Schwarz à présenter le budget 2013 de la CdG (proposition $n^{\circ} 1$ au point 4.2 du Comité central).

\subsection{Budget 2013 de la CdG}

Roland Schwarz/CdG communique le budget de la CdG. Il se tient à disposition pour d'éventuelles questions.

J. de Haller donne la parole à Emanuel Waeber/ $F M H$ qui présente le budget de la FMH (proposition $\mathrm{n}^{\circ} 1$ au point $\mathrm{n}^{\circ} 4.3$ du Comité central).

\subsection{Budget 2013 de la FMH}

Emanuel Waeber indique que la FMH a procédé à quelques adaptations budgétaires suite à la décision de la dernière Chambre médicale du 7 juin dernier de revoir entièrement le processus budgétaire et d'approuver le budget de l'année suivante désormais à la séance d'automne et non plus du printemps. Toutefois, pour des raisons de calendrier et de technique financière, le processus de planification et de budgétisation nouvellement initié ne déploiera ses effets que dans un an avec la présentation du budget 2014. Le budget 2013 présente une perte de CHF 381000.- au total, soit CHF 256000.- pour la FMH et CHF 125000.- pour l'ISFM. Les chiffres donnés pour les pertes en 2013 doivent être considérés avec prudence car le résultat budgétisé ne tient pas encore compte de la précision du nouveau processus de budgétisation.

Les comptes trimestriels révisés donnent la possibilité de présenter un «forecast» (budget prévisionnel) aux membres de la Chambre médicale. Conformément au budget 2013, les recettes de la FMH s'élèvent à 20,5 millions de francs et les dépenses liées à environ 19,65 millions, les charges pour projets à environ 1,1 million. Avec le forecast, la FMH vise à atteindre davantage de fiabilité et de transparence dans la structure des coûts.

Le 7 juin 2012,E. Waeber avait présenté une perte de CHF 441492.- pour 2013. Suite à la décision de la dernière Chambre médicale de déplacer l'approbation du processus budgétaire à la séance de décembre, on a encore procédé à des économies internes supplémentaires. Grâce aux bons résultats d'exploitation 2011, aucune dissolution de réserves supplémentaire n'est nécessaire.

Le président donne la parole à Ernst Gähler/CC pour la contribution spéciale NAKO (proposition $\mathrm{n}^{\circ} 1$ au point 4.3.1 du Comité central).

\subsubsection{Contribution spéciale NAKO}

Ernst Gähler/CC communique que la FMH perçoit auprès des membres des catégories 1 et 2 une contribution spéciale liée de 40 francs en faveur de l'organe national de consolidation NAKO qui collecte les données de tous les centres fiduciaires (NewIndex). E. Gähler souligne combien ces données sont importantes pour le corps médical. Elles sont précieuses non seulement pour la comparaison entre collègues ou pour les procédures EAE, mais aussi pour les négociations tarifaires dans le cadre du projet TARVISION ou de la révision de la liste des analyses. Grâce au NAKO, la FMH est la seule organisation professionnelle de la santé à disposer constamment de chiffres consolidés. A cette occasion, Ernst Gähler rappelle également l'importance des données ROKO sans lesquelles les modèles de coûts ne pourraient pas être actualisés. Pour cette raison, il lance un appel à tous les médecins en cabinet privé de livrer leurs données ROKO et il invite les sociétés cantonales de médecine et les sociétés de discipline à le rappeler à leurs membres. 


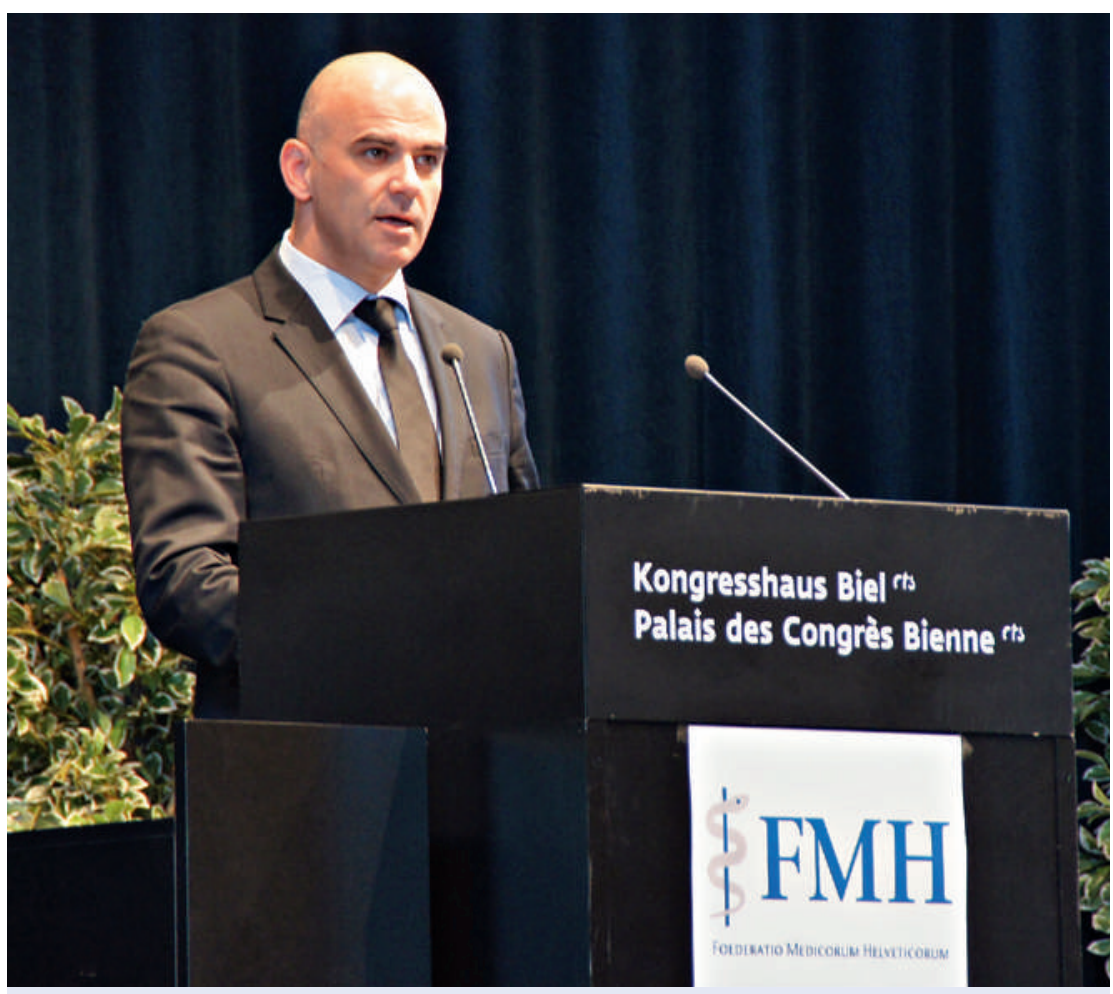

Alain Berset, conseiller fédéral et chef du DFI, place également le bien des patientes et des patients au centre de sa politique.

Pourquoi demander une contribution spéciale? Le financement du NAKO n'est pas garanti par les conventions passées avec les organisations médicales alors que le système NAKO doit être de plus en plus performant et que le corps médical a besoin de toujours plus de données consolidées pour les négociations. Mais les adaptations organisationnelles en cours devraient permettre de transférer la contribution NAKO dans le budget ordinaire de la FMH dès 2014.

- Les propositions sur l'approbation du budget $\left(n^{\circ} 1\right.$ au point $n^{\circ} 4.1 ; n^{\circ} 1$ au point $n^{\circ} 4.2, n^{\circ} 1$ au point $n^{\circ} 4.3$ et $n^{\circ} 1$ au point $n^{\circ} 4.3 .1$ ) sont acceptées à une large majorité, sans opposition et par deux abstentions.

\section{Autres modifications des Statuts, du Règlement d'exécution et du Code de déontologie}

5.1 Modification des Statuts - Mise en œuvre de la proposition Girr (ZH) présentée à la Chambre médicale de mai 2011

\subsubsection{Modification des Statuts (proposition du Comité central)}

5.1.2 Modification des Statuts (proposition AMG) Jacques de Haller donne la parole à Hanspeter Kuhn/ FMH afin qu'il présente la proposition du Comité central pour la mise en œuvre de la proposition A. Girr/ZH acceptée par la Chambre médicale de mai
2011 (proposition $\mathrm{n}^{\circ} 1$ au point $\mathrm{n}^{\circ}$ 5.1.1). H.P. Kuhn en rappelle le contenu: «Le Comité central représente auprès du grand public la position de la majorité des membres sur les questions relatives à notre profession. Si cette position est contestée au sein du corps médical, il convient de procéder à une enquête auprès des membres.» Avec cette proposition, A. Girr a tenu à s'assurer que le Comité central sache bien ce qu'il a à représenter sur le plan politique. Simultanément, il s'agit aussi de veiller à ce que des votations générales ne soient organisées qu'au moment où elles sont vraiment nécessaires. H.P. Kuhn communique que le Comité central propose à la Chambre médicale la formulation suivante (complétant l'article 49 des Statuts de la FMH, alinéa 3): «Si la position officielle de la FMH dans des questions de politique professionnelle ne reflète pas clairement l'opinion de la base, le Comité central peut organiser une enquête auprès des membres de la FMH.» H.P. Kuhn explique que c'est toujours une question d'appréciation si un avis est clair ou s'il ne l'est pas. Il ajoute que la politique, c'est l'art de prendre des décisions: quand faut-il demander un avis? Quand faut-il simplement avancer? Le Comité central a déjà procédé de son propre chef à des enquêtes auprès des membres et il est déjà possible, en vertu des Statuts, pour la Chambre médicale ou l'Assemblée des délégués de demander au Comité central de réaliser une telle enquête. Le Comité central n'est donc pas le seul à décider si la volonté de la base est suffisamment connue. La mention explicite de cette possibilité dans les compétences du CC a toutefois pour avantage que celle-ci est désormais présentée de manière transparente pour tous.

Didier Châtelain/GE présente, au nom de l'Association des médecins du canton de Genève, la proposition $n^{\circ} 1$ au point $n^{\circ} 5.1$, qui demande une formulation bien plus contraignante:

Article 49 alinéa 3: Avant de prendre une position publique engageant la FMH sur des questions d'ordre national relatives à la politique professionnelle, le CC doit procéder à une enquête auprès de ses membres pour connaitre l'opinion de la base. Le CC doit renouveler une telle enquête si les circonstances ont changé.

Marc Müller/Médecins de famille Suisse s'exprime résolument contre la proposition de l'Association des médecins du canton de Genève, qui va trop loin, à son avis. Si cette proposition est acceptée, la FMH peut supprimer tous ses organes, à l'exception du Comité central et de l'enquête auprès des membres. Thomas Heuberger/BE le soutient et indique que l'on doit prendre garde à ne pas paralyser la FMH avec des enquêtes qui se répètent. Angelo Cannova/ZH n'est pas de cet avis et il estime qu'on n'abusera pas de cet instrument mais qu'il est indiqué de connaître l'opinion de la base lors de décisions importantes. Christine Romann/CC précise que la proposition des Genevois porte sur des «questions en lien avec la politique professionnelle». Elle concerne donc presque tout ce que fait le Comité central. Les Genevois demandent 
ici de remettre en question la structure de la FMH. $D$. Châtelain/GE considère que cette crainte est exagérée et il fait remarquer que le travail du Comité central aurait été simplifié ces deux dernières années si l'on avait mieux connu l'opinion de la base.

Le président passe aux votes.

- La proposition $n^{\circ} 1$ au point $n^{\circ} 5.1 .2$ de l'Association des médecins du canton de Genève est rejetée par 88 voix contre, 9 voix pour et 5 abstentions.

- La proposition $n^{\circ} 1$ au point $n^{\circ}$ 5.1.1 du Comité central est clairement acceptée par la majorité.

5.2 Adaptation du Règlement d'exécution: art. 31 «Processus de planification et de budgétisation» Jacques de Haller donne la parole à Anne-Geneviève Bütikofer/FMH afin qu'elle présente la proposition $\mathrm{n}^{\circ} 1$ au point $\mathrm{n}^{\circ} 5.2 \mathrm{du}$ Comité central concernant le Règlement d'exécution de la FMH et le processus de planification et de budgétisation. A.-G. Bütikofer explique qu'il s'agit ici seulement d'une formalité. Comme la dernière Chambre médicale a décidé de se réunir deux fois par an pour optimiser entre autres le processus budgétaire, il s'agit maintenant d'adapter le Règlement d'exécution en conséquence. Désormais, le budget sera adopté à la séance d'automne de la Chambre médicale et non plus à celle du printemps.

- La proposition $n^{\circ} 1$ au point $n^{\circ} 5.2$ est approuvée à une large majorité.

\section{Election complémentaire pour la vice-présidence de la Commission de déontologie de la FMH}

La SMSR propose d'élire Charles-Abram Favrod-Coune pour remplacer Alexandre Marmier décédé. Il est élu à la majorité, sans opposition ni abstention.

\section{Confirmation de nouveaux délégués à l'Assemblée des délégués}

\subsection{Délégués ordinaires/délégués suppléants}

Le président rappelle que les délégués concernés ont été élus par les organisations professionnelles et que leur élection est soumise à la Chambre médicale pour confirmation. Il demande si quelqu'un désire prendre la parole. Comme ce n'est pas le cas, Jacques de Haller passe au vote.

\section{Confirmation de délégués ordinaires}

SMSR

- Jusqu'ici: Charles-Abram Favrod-Coune et JeanDaniel Schumacher.

- Désormais: Véronique Monnier-Cornuz et MarcHenri Gauchat

SFSM

- Jusqu'ici: Christian Seiler

- Désormais: Urs Kaufmann

VEDAG

- Jusqu'ici: vacant (jusqu'au 7.6.2012: Urs Stoffel)
- Désormais: Karin Stadlin

ASMAC

- Jusqu'ici: Fabrice Dami et Vincent Cattin

- Désormais: Hervé Spechbach et Anja Zyska

Confirmation de délégués suppléants:

SMSR

- Jusqu'ici: Marc-Henri Gauchat

- Désormais: Jean-Daniel Schumacher

Le président propose à la Chambre médicale de voter en bloc.

- La Chambre médicale confirme sans opposition ni abstention l'élection des délégués ordinaires et des délégués suppléants présentés ci-avant.

\section{Divers}

8.1 Projet d'article 55a LAMal (réintroduction temporaire de l'admission selon le besoin)

Jacques de Haller passe à la proposition $\mathrm{n}^{\circ} 1$ au point $\mathrm{n}^{\circ} 8.1$ de la Société vaudoise de médecine (SVM). Celle-ci a la teneur suivante: «Au cas où l'article 55 a serait adopté par le parlement fédéral, alors il devrait être appliqué aux médecins praticiens.». Le président donne la parole à Véronique Monnier-Cornuz/SVM. A son avis, il faut absolument empêcher, en cas de réintroduction de la clause du besoin, que des médecins praticiens puissent s'établir et ouvrir un cabinet sans contrôle. Concernant les médecins praticiens, il s'agit souvent de médecins dépourvus d'une formation correcte, sans formation postgraduée ni examen final. D'autres par ailleurs, des spécialistes, viendraient de l'étranger pour s'établir comme médecins praticiens. Il s'agit de protéger les jeunes médecins suisses qui sont tous au bénéfice d'une bonne formation. V. Monnier-Cornuz souhaite que la Chambre médicale émette un signal à l'intention des politiques.

J. de Haller précise que les spécialistes suisses comme les généralistes ou les internistes disposant d'un deuxième titre n'ont pas la possibilité de renoncer à leurs titres alors que des médecins venus de l'étranger pourraient simplement indiquer qu'ils sont médecins praticiens sans titre dans le but de contourner la clause du besoin. J. de Haller rappelle également que la Chambre médicale n'est plus l'instance politique de la FMH mais que cette compétence revient à l'Assemblée des délégués. La Chambre médicale peut toutefois adopter une résolution à l'intention du Comité central et de l'Assemblée des délégués.

Pierre-Alain Schneider/GE propose que la Chambre médicale demande d'étendre la clause du besoin à tous les médecins. Cela répondrait à un besoin réel, et pas seulement dans le canton de Genève. MarcHenri Gauchat/SMSR précise que les médecins généralistes qui viennent de France pour s'établir en Suisse romande ne disposent pas d'une formation comparable à celle de leurs collègues suisses. La 
majorité d'entre eux n'ont jamais travaillé dans un hôpital. En revanche, les médecins allemands peuvent attester d'une formation similaire. Vincenzo Liguori/TI indique que le Tessin soutient les collègues de la Suisse romande, c'est une région frontalière qui est confrontée aux mêmes problèmes. Ce n'est pas schizophrène de critiquer, d'une part, le manque de médecins de famille et de vouloir, d'autre part, stopper le flux de médecins en provenance de l'étranger. Les collègues étrangers ne vont pas ouvrir leur cabinet là où il en manque, par exemple dans les régions de montagne, mais dans les villes où la pénurie se fait le moins ressentir. Beat Gafner/BE souligne qu'il s'oppose à la clause du besoin parce qu'il ne veut pas qu'un groupe de collègues, qui à ses yeux ont fait leurs preuves, soit exclu pour les dix prochaines années. Pour cette raison, il ne soutient pas la proposition de la Société vaudoise de médecine. La solution doit venir au niveau cantonal. Marc Müller/Médecins de famille Suisse estime que vouloir appliquer la clause du besoin aux médecins de premier recours est problématique, alors qu'il en manque de toute évidence. A son avis, cette réglementation serait encore plus impensable que la prolongation de la clause du besoin déjà combattue par les médecins de famille. Pour eux, il faut réguler, vers le bas et vers le haut, mais il ne faut pas d'interdiction.

Le président passe au vote de la proposition de la Société vaudoise de médecine et de la proposition complémentaire des médecins genevois demandant que la Chambre médicale se prononce en faveur d'une extension de la clause du besoin aux médecins de premier recours.

- La proposition $n^{\circ} 1$ au point $n^{\circ} 8.1$ de la Société vaudoise de médecine est acceptée à une large majorité et quelques oppositions.

- La proposition de Pierre-Alain Schneider/GE est rejetée par 61 voix contre 54 et 9 abstentions.

\section{Calendrier}

La prochaine Chambre médicale aura lieu jeudi 25 avril 2013 au Palais des congrès de Bienne. Jacques de Haller remercie les délégués de leur participation active et clôt la séance à $16 \mathrm{~h} 30$.

\begin{tabular}{|c|c|c|c|}
\hline \multicolumn{4}{|c|}{ Glossaire } \\
\hline AD & Assemblée des délégués & LAMal & Loi sur l'assurance-maladie \\
\hline \multirow[t]{2}{*}{ AMG } & \multirow{2}{*}{$\begin{array}{l}\text { Association des Médecins du } \\
\text { canton de Genève }\end{array}$} & MFE & Médecins de famille Suisse \\
\hline & & NAKO & Centre de consolidation nationale \\
\hline \multirow[t]{2}{*}{ ASMAC } & \multirow{2}{*}{$\begin{array}{l}\text { Association suisse des médecins- } \\
\text { assistant(e)s et chef(fe)s de clinique }\end{array}$} & OFSP & Office fédéral de la santé publique \\
\hline & & \multirow[t]{2}{*}{ ОМст } & \multirow{2}{*}{$\begin{array}{l}\text { Ordine dei Medici } \\
\text { del Cantone Ticino }\end{array}$} \\
\hline BMS & Bulletin des médecins suisses & & \\
\hline CC & Comité central & \multirow[t]{2}{*}{ ReMed } & \multirow{2}{*}{$\begin{array}{l}\text { Réseau de soutien } \\
\text { pour les médecins }\end{array}$} \\
\hline CdG & Commission de gestion & & \\
\hline \multirow[t]{2}{*}{ CDS } & \multirow{2}{*}{$\begin{array}{l}\text { Conférence suisse des directrices } \\
\text { et directeurs cantonaux de la santé }\end{array}$} & ROKO & Etude permanente des coûts \\
\hline & & \multirow[t]{2}{*}{ SFSM } & \multirow{2}{*}{$\begin{array}{l}\text { Swiss Federation of Specialities } \\
\text { in Medicine }\end{array}$} \\
\hline ChM & Chambre médicale & & \\
\hline СTM & Commission des tarifs médicaux & \multirow[t]{2}{*}{ SMSR } & \multirow{2}{*}{$\begin{array}{l}\text { Société médicale de la Suisse } \\
\text { romande }\end{array}$} \\
\hline DFI & Département fédéral de l'intérieur & & \\
\hline EAE & Efficacité, adéquation, économicité & \multirow[t]{2}{*}{ SSMI } & \multirow{2}{*}{$\begin{array}{l}\text { Société suisse de médecine } \\
\text { interne générale }\end{array}$} \\
\hline \multirow[t]{2}{*}{$\mathrm{fmCh}$} & \multirow{2}{*}{$\begin{array}{l}\text { Foederatio medicorum } \\
\text { Chirurgicorum helvetica }\end{array}$} & & \\
\hline & & SVM & Société vaudoise de médecine \\
\hline FMH & Fédération des médecins suisses & \multirow[t]{2}{*}{ TARMED } & \multirow{2}{*}{$\begin{array}{l}\text { Tarif à l'acte pour les prestations } \\
\text { médicales ambulatoires }\end{array}$} \\
\hline GRAT & Révision complète du tarif médical & & \\
\hline $\mathrm{H}+$ & Les hôpitaux de Suisse & TARVISION & $\begin{array}{l}\text { Projet de la FMH pour la révision } \\
\text { de la structure tarifaire du TARMED }\end{array}$ \\
\hline HPC & Carte de professionnel de santé & \multirow{3}{*}{$\begin{array}{l}\text { TransAL } \\
\text { VEDAG }\end{array}$} & \multirow{3}{*}{$\begin{array}{l}\text { Projet relatif à la liste des analyses } \\
\text { Association des sociétés médicales } \\
\text { de la Suisse alémanique }\end{array}$} \\
\hline IKS & Service de contrôle interne & & \\
\hline ISFM & $\begin{array}{l}\text { Institut suisse pour la formation } \\
\text { médicale postgraduée et continue }\end{array}$ & & \\
\hline
\end{tabular}

